\title{
An Energy-efficient Routing Algorithm for Disruption Tolerant Networks
}

\author{
Yukun Yao, Wenxi Zheng, Zhi Ren \\ Chongqing Key Lab of Mobile Communications Technology \\ Chongqing University of Post and Communications \\ Chongqing, 400065, China \\ e-mail: yaoyk@cqupt.edu.cn
}

\begin{abstract}
In Disruption Tolerant Networks (DTN), information is exchanged between mobile nodes with the same transmit power. It is an energy-consuming process and highlights the need for energy-efficient power control mechanisms. If the transmit power of nodes is too low, they might miss many of the opportunities of transmission. On the other hand, high transmit power is energy inefficient. In this paper, we propose ERBC (Energy-efficient Routing algorithm Based on Crosslayer design), an energy-efficient routing algorithm based on cross-layer design. Instead of using fixed transmit power, ERBC dynamically chooses the transmit power using both the cross-lay design and the RSSI technology. Meanwhile, ERBC broadcast data packets partially in order to enhance the delivery ratio. Through simulation, we demonstrate that ERBC can reduce $37 \%$ for per-bit energy consumption than that in basic epidemic routing protocol.
\end{abstract}

Keywords-Disruption Tolerant Networks(DTN); epidemic routing; Power control; RSSI

\section{INTRODUCTION}

Previously, the immediately, anytime, anywhere access to the information had been the main aspects of networking research. However, because of the disconnected wireless links, many researchers have been in interested in a new class of applications that are tolerant to disruption recently. The DTN is converted from the mobile Ad-hoc networks. Information is exchanged opportunistically between nodes when they are with in communication range of each other and does not need complete links between source-destination pairs $^{[1]}$.

Most of the mobile nodes are deployed in the complex situations and powered by batteries which lead to the difficulties of replacing and charging the batteries. There are researches ${ }^{[2]}$ show, in general, the transmitting and receiving signals are a part of the most energy consuming in wireless networks. However, if each node transmits packets to its neighbor with the maximum power, its battery will be used up quickly.

The rest of this paper is organized as follows: first the related works are discussed in section II . In section III, we describe the energy-efficient routing algorithm based on Epidemic routing in detail. In section IV, we evaluate the energy saving performance of ERBC and basic epidemic routing. And section $\mathrm{V}$ is the conclusion of the whole paper.

\section{RELATED WORK}

At present, there are lots of researches on DTN routing algorithms, and have gained rich achievements in this field $^{[3][4][5]}$. However, little attention is paid to the power control of DTN. Because the frequent contact probing is energy inefficient, $\mathrm{STAR}^{[6]}$ a contact-probing algorithm is proposed. Instead of using constant probing intervals, it uses both the short-term contact history and the long-term contact history based on time of day information, and then dynamically chooses the probing interval to achieve the purpose of energy-saving. The efficient algorithms and policies are crucial, so reference ${ }^{[7]}$ maximizes the message delivery probability, meanwhile reduces the delivery cost for opportunistic forwarding. And they demonstrate that the performance of the threshold dynamic policy is the best among the static and continuous dynamic policies. The node's batteries will be used up quickly if it transmits packets to all its encounters. To address this issue, an nepidemic routing algorithm ${ }^{[8]}$ is proposed. In this algorithm, each node transmits data packets only when the number of neighbors reaches a certain threshold. The experiment shows that the delivery performance of n-epidemic routing algorithm can increase $434 \%$ average than that in the basic Epidemic Routing.

There are lots of research on power control in Ad-hoc networks and sensor networks. PARO ${ }^{[9]}$ is a dynamic power control routing algorithm which can minimize the transmit power needed to forward packets between wireless nodes. In order to reduce the aggregate transmit power consumed, one or more intermediate nodes are elected to forward packets on behalf of source-destination pairs, and it can be appropriate for a number of networking environments. There is a routing protocol ${ }^{[10]}$ for point-to-point communications using directional antennas on the nodes. It can maximize the utilization of the limited energy available with the sensor nodes. This algorithm not only heals the network partitions, but can also achieve high throughput and fairness across different flows, in addition to maximizing the network lifetime.

\section{ENERGY-EFFICIENT ROUTING ALGORITHM BASED ON CROSS-LAYER DESIGN}

We combine with the basic principles of the Epidemic Routing algorithm. To address the issue of using fixed transmit power to exchange the information causing the unnecessary energy consumption, and in the wireless 
communication network, the sending data packets mode can result in the nodes energy waste within the same communication range, we combine with the basic principles of the Epidemic Routing algorithm and propose ERBC, an energy-efficient routing algorithm based on cross-layer design. It predicts the distance between two nodes reasonably by the RSSI techniques then selects the minimal transmit power level to transmit the packets. Then ERBC broadcast data packets in special case. The case is Have multi-neighbors $\}$ \{No destination node in the neighborhood $\} \cap\{$ Neighbor nodes don't storage these data packets $\}$. It can enhance the spread of data packets in the entire network, and decrease the number of data packets sending. All in all, we can achieve the purpose of energysaving.

Because using RSSI does not need to increase extra devices and extra energy consumption, relatively speaking, the cost and complexity are lower. It has been widely used in many kinds of wireless multi-hop networks, but rarely used in the routing algorithms. ERBC obtains the distance between two nodes by RSSI. And after a reasonable estimate it chooses the proper transmit power level to send packets. At the same time, the network layer should inform MAC sublayer and physical layer by the cross-layer technique, then adjust the transmit power for SV packets and data packets. The cross-layer approach is shown in Fig. 1.

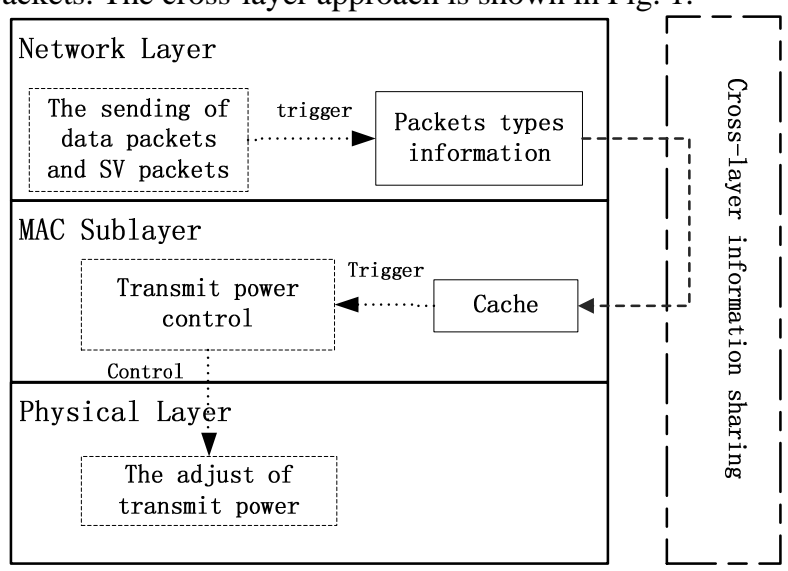

Figure 1. Cross-layer approach

For the energy consumption of transmit power adjustment mechanism of ERBC, we give the following lemma.

\section{A. lemma and proof}

Lemma 1: In the same network conditions, the average transmit power of ERBC is smaller than that in Epidemic Routing algorithm.

Proof: Consider the network with $\mathrm{N}$ nodes. A node $\mathrm{i}$ is located in the centre of a circle with the radius $\mathrm{R}$ which is equal to the communication range. Its neighbor $\mathrm{j}$ is located at any point of this circle area. The distance of the two nodes is represented by $\mathrm{x}$, and the probability density function of $\mathrm{x}$ is calculated as follows.

$$
f(x)= \begin{cases}\frac{2 x}{R^{2}}, & 0<x<R \\ 0, & \text { otherwise }\end{cases}
$$

Radius $\mathrm{R}$ is divided into $\mathrm{n}$ equal parts. Each part corresponds to the transmit power level. The relationship between the transmit power level $\mathrm{y}$ and the distance $\mathrm{x}$ is described as follows.

$$
y_{\mathrm{ERBC}}=\left\lceil\frac{n x}{R}\right\rceil
$$

Therefore, the mathematical expectation of the power level y is computed as follows.

$$
E(Y)_{\mathrm{ERBC}}=\sum_{i=1}^{n} i \int_{(i-1) R / n}^{i R / n} f(x) d x
$$

Combined with equation (3), we can get the following value.

$$
E(Y)_{\text {REBC }}=\frac{2 n}{3}-\frac{1}{6 n}+\frac{1}{2}
$$

The mathematical expectation of the fixed transmit power of the Epidemic Routing is computed as follows.

$$
E(Y)_{\text {Epidenic }}=n
$$

After the mathematical analysis, if the transmit power level is larger than one, then we have:

$$
E(Y)_{\text {ERCPC }}<E(Y)_{\text {Epidemic }}
$$

In other words, the average transmit power of ERBC is smaller than the Epidemic Routing algorithm's. The proof is finished.

\section{B. Detailed operations}

Step 1. In ERBC algorithm, nodes need to periodically broadcast Hello packets (1-hop) from their network layers. The packets include its network address.

Step 2. If its neighbor node receives a Hello packet, it should check the time interval of recently received two Hello packets. If the time interval is smaller than the threshold, we consider that it's the new neighbor. Then calculate the distance $d_{\text {Hello }}$ of two nodes by the RSSI, and predict the minimal transmit power to send the SV packets by $d_{\text {Hello }}$. In order to ensure the success of the delivery of the SV packets, we assume that the neighbor node uses the maximum speed, and the direction of motion is opposite, just like the following equation.

$$
\begin{aligned}
d_{\mathrm{SV}}= & d_{\text {hello }}+T_{\text {max }}\left(V_{\text {max }}+V_{\text {mine }}\right) \\
& +\frac{\left(L_{\text {hello }}+2 L_{\mathrm{SV}}\right) V_{\text {max }}+L_{\mathrm{SV}} V_{\text {mine }}}{B}
\end{aligned}
$$

Here, the $T_{\max }$ indicates the maximum data processing delay of nodes. The $V_{\max }$ indicates the maximum speed of nodes. The $V_{\text {mine }}$ indicates its own speed. The $L_{\text {hello }}$ and $L_{\mathrm{SV}}$ indicate packets length of Hello packets and SV packets in bit, respectively. $\mathrm{B}$ indicates the bandwidth. The $d_{\mathrm{SV}}$ corresponds with a transmit power. We can select one of the most suitable transmit power level to deliver SV packet.

Step 3. When its neighbor receives a SV packet, it checks out the data packets that are not stored but counterpart node stored by comparing its own packet indexes. Then it loads 
the indexes of these packets into a request packet and sends it to the counterpart node use the initial transmit power.

Step 4. The counterpart node can receive the request packet, it calculates the current distance $d_{\text {request }}$ between the two nodes. Accounting to the indexes of the request packet, it delivers data packets to counterpart node. If the neighbor node is the destination of data packets, then we transmit them directly; otherwise broadcast them. The setting of the transmit power level is determined by the $d_{\text {hello }}$ and $d_{\text {request }}$. And it has the following three conditions.

1) $d_{\text {hello }}>d_{\text {request }}$

It demonstrates that two nodes are approaching to each other, and maintain this status in short time. So the communication range of the data packets is $d_{\text {request }}$.

2) $d_{\text {hello }}=d_{\text {request }}$

It shows that two nodes are relatively static, and maintain this status in short time. Sets $d_{\text {data }}=d_{\text {request }}$.

3) $d_{\text {hello }}<d_{\text {request }}$

It shows that two nodes are keeping away from each other, and maintain this status in short time. We also assume the counterpart node uses the maximum speed and the direction of motion is opposite. So we have:

$$
\begin{aligned}
d_{\text {data }}= & d_{\text {request }}+T_{\text {max }}\left(V_{\text {max }}+V_{\text {mine }}\right) \\
& +\frac{\left(L_{\text {request }}+2 L_{\text {data }}\right) V_{\text {max }}+L_{\text {data }} V_{\text {mine }}}{B}
\end{aligned}
$$

Here, the $L_{\text {request }}$ and $L_{\text {data }}$ indicate packets length of request packets and data packets in bit, respectively.

\section{SIMULATION ANALYSIS}

\section{A. Setting of key parameters}

1) Per-bit energy consumption: it is the average energy consumption which means the energy of 1 bit data sending from source to destination and it is presented as Ebit. So we set the Ebit as follows.

$$
E_{\text {bit }}=\frac{\sum_{k=1} E_{\text {transk }}+\sum_{k=1} E_{\text {Rxk }}}{N_{\text {bit }}}
$$

Here $E_{\text {transk }}$ and $E_{\mathrm{Rxk}}$ are the energy consumption of receiving and transmission of node $\mathrm{K}$. $N_{\text {bit }}$ indicates the number of data packets in bit which successfully reached the destination.

2) Network lifetime: it is an important indicator of network performance. In the simulation, we define the experience time of the first death node as the network lifetime 错误!未找到引用源。.

3) Success rate: divide the number of data packets which have successfully reached the destination by the total number of data packets. It is calculated as follows.

$$
D_{\text {rate }}=\frac{\sum_{k=1} D_{k}}{\sum_{m=1} S_{m}} \times 100 \%
$$

Here $D_{\mathrm{k}}$ indicates the number of data packets which have reached the destination. $S_{\mathrm{m}}$ indicates the number of data packets generated by the source node.

\section{B. Simulation environments and parameters}

We use the OPNET14.5 simulation software to simulate and model. There are 100 mobile nodes evenly and randomly spread over an area of $1500 \mathrm{~m} \times 600 \mathrm{~m}$. The initial communication range $R \in\{10,25,50,75,100\}(m)$. Depending on the different $\mathrm{R}$, we set five different simulation scenarios. The moving nodes using the random waypoint mobility model with a speed uniformly distributed between $1-19 \mathrm{~m} / \mathrm{s}$, and the pause time is $0 \mathrm{~s}$. We randomly select 60 nodes as sources and destinations. Each node sends data packets to other 59 nodes with $1 \mathrm{~s}$ of the transmission interval. The other 40 nodes only forward the data packets. The table 1 shows the main parameters used in simulation.

\begin{tabular}{cc} 
TABLE I. & THE MAIN PARAMETERS IN SIMULATION \\
\hline Variable & Value \\
\hline The initial energy of nodes & $10 \mathrm{~J}$ \\
The stander of MAC sublayer and PHY. layer & IEEE802.11a \\
The rate of data transfer & \\
The simulation time & $54 \mathrm{Mbps}$ \\
Transmit power levels & $5000 \mathrm{~s}$ \\
& 8
\end{tabular}

The communication range of node energy levels is shown

\begin{tabular}{|c|c|}
\hline $\begin{array}{c}\text { the initial } \\
\text { communication } \\
\text { range of nodes/m }\end{array}$ & $\begin{array}{l}\text { the communication range of node } \\
\text { energy levels } / \mathrm{m}\end{array}$ \\
\hline 10 & $10,9,8,7,6,5,4,3$ \\
\hline 25 & $25,22 、 19,16,13,10,7,4$ \\
\hline 50 & $50 、 44,38,32,26,20 、 14,8$ \\
\hline 75 & 75, 66,57,48,39,30,21, 12 \\
\hline 100 & $100,85,70,55,40,25,10,5$ \\
\hline
\end{tabular}
in Table II .

TABLE II. THE COMMUNICATION RANGES OF NODE ENERGY LEVELS

C. Simulation results and analysis

1) Per-bit energy consumption

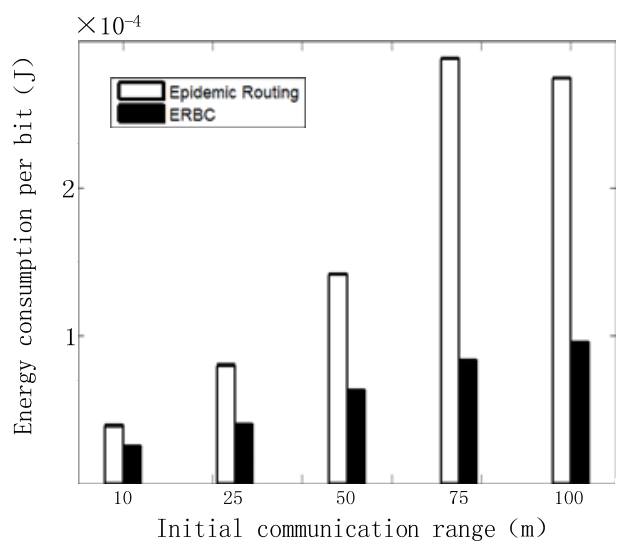

Figure 2. The comparison of per-bit energy consumption

All the energy consumption of data frames and control frames of sending and receiving is divided by the number of data packets bit which successfully reach the destinations. 
Fig. 2 shows that ERBC algorithm is better than Epidemic Routing algorithm in five scenarios. If the communication range of nodes is $100 \mathrm{~m}$, ERBC algorithm has an obvious effect of energy saving. Because it has a large interval between levels, and more neighbor nodes can receive the broadcast packets. Whereas if the communications range of nodes are $10 \mathrm{~m}$, the energy saving effect is not obvious.

2) The network lifetime

Fig. 3 shows that compared with Epidemic Routing algorithm, the network lifetime has improved in five scenarios. When the initial communication range is $10 \mathrm{~m}$, there is no node death in the simulation time of 5000s.

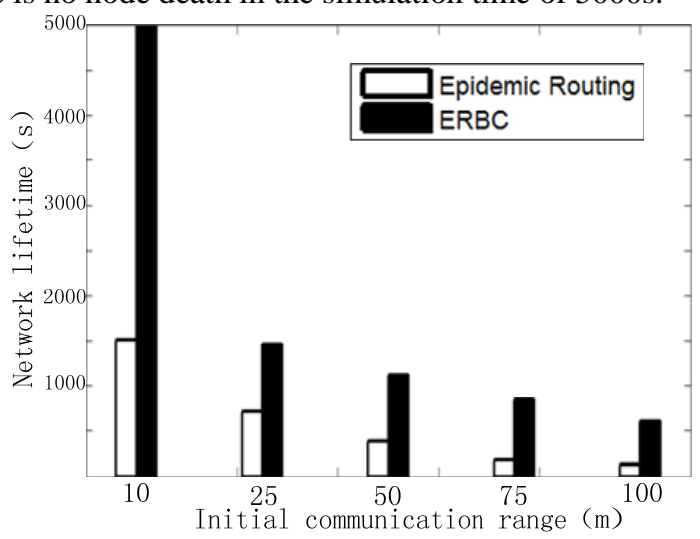

Figure 3. The comparison of network lifetime

3) The success rate of data packets transmission

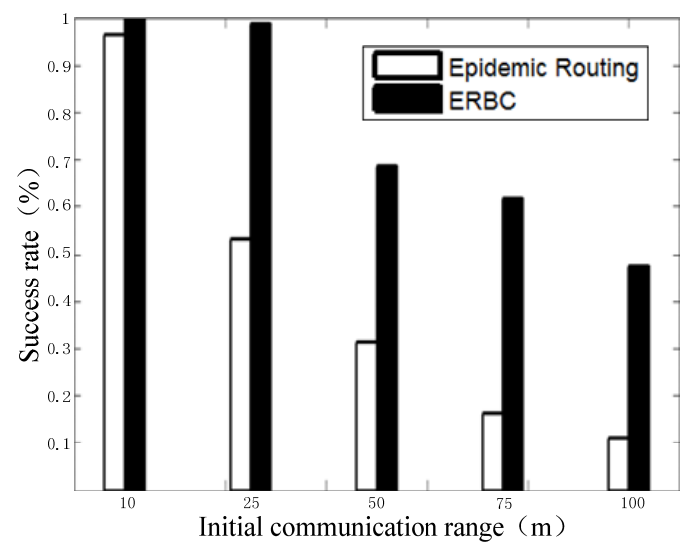

Figure 4. The comparison of success rate

Fig. 4 shows that the success rate of ERBC algorithm is higher than that in Epidemic Routing algorithm. The success rate of ERBC algorithm is $100 \%$ in the scenario of $10 \mathrm{~m}$, with the expansion of communication range the success rate decreases, because the nodes can survive long in a small communication range; contrary, the more nodes will dead, so success rate is the lowest in the $100 \mathrm{~m}$ communication range.

\section{CONCLUSIONS AND FUTURE WORK}

In this paper we propose an energy-efficient routing algorithm in DTN-ERBC. It can protect the energy of nodes and prolong the network lifetime by the cross-layer design, power control and broadcasting data packets. Its performance and effectiveness have been verified in the theoretical analysis and simulation results. In the future, we will expand the energy-saving of ERBC to the fields of buffer management and node sleep in order to build the green DTN with a longer lifetime.

\section{ACKNOWLEDGMENT}

This work was supported in part by the National Natural Science Foundation of China under Grant No. 60972068, the Scientific Research Starting Foundation for Returned Overseas Chinese Scholars, Ministry of Education of China under Grant No. 2010-1561, the open project of Emergency Communication Laboratory of Chongqing under Grant No. 201201, and the special fund of Chongqing key laboratory (CSTC) under Grant No. D2011-24.

\section{REFERENCE}

[1] L. Pelusi, A. Passarella, M. Conti. Opportunistic networking: data forwarding in disconnected mobile ad hoc networks. IEEE Communications Magazine, Vol. 44, No. 11, 2006:134-141.

[2] A. Vahdat, D. Becker. Epidemic Routing for Partially Connected Ad Hoc Networks. Tech. Rep. CS-2000-06, Department of Computer Science, Duke University, Durham, NC, 2000.

[3] P. Gilbert, V. Ramasubramanian, P. Stuedi, et al. Peer-to-Peer Data Replication Meets Delay Tolerant Networking[C]. Proceedings of the 31st International conference on Distributed Computing Systems, Minneapolis, 2011:109-120.

[4] S. Medjiah, T. Ahmed. Orion Routing Protocol for Delay-Tolerant Networks[C]. Proceedings of IEEE International Conference on Communications, Kyoto, 2011: 1-6.

[5] Y. Zhu, B. Xu, X.H. Shi, et al. A Survey of Social-based Routing in Delay Tolerant Networks: Positive and Negative Social Effects[J]. IEEE Communications Surveys \& Tutorials, 2012, 14(2): 1-15.

[6] W. Wang, M. Motani, V. Srinivasan. Opportunistic energy-efficient contact probing in delay-tolerant applications[J]. IEEE/ACM Transactions on Network, 2009, 17(5): 1592-1605.

[7] Y. Li, Y.R. Jiang, D.P. Jin, et al. Energy-Efficient Optimal Opportunistic Forwarding for Delay-Tolerant Networks[J]. IEEE Transactions on Vehicular Technology, 2010, 59(9): 4500-4512.

[8] X. Lu, P. Hui. An Energy-Efficient n-Epidemic Routing Protocol for Delay Tolerant Networks[C]. Proceedings of the 5th International Conference on Networking, Architecture, and Storage, Macau, China, 2010: 341-347.

[9] J. Gomez, A. T. Campbel, M. Naghshineh, et al. PARO: supporting dynamic power controlled routing in wireless Ad hoc networks[J]. Wireless Networks, 2003, 9(5): 443-460.

[10] K. Selvaradjou, N. Handigol, A. A. Franklin, et al. Murthy Energyefficient directional routing between partitioned actors in wireless sensor and actor networks[J]. IET Communications, 2010, 4(1): 102115. 